\title{
Modelling extrasolar planetary atmospheres
}

\author{
France Allard \\ Centre de Recherche Astrophysique de Lyon, \\ UMR 5574: CNRS, Université de Lyon, École Normale Supérieure de Lyon, \\ 46 allée d'Italie, F-69364 Lyon Cedex 07, France \\ email: fallard@ens-lyon.fr
}

\begin{abstract}
The atmospheres of close-in Extrasolar Giant Planets (EGPs) experience important stellar radiation, raising the question of the heat redistribution around the planetary surface and of the importance of photochemistry effects for their spectral properties. They experience mass loss via quasi-thermal escape of their lightest elements. They rotate and experience tidal effects. Model atmospheres struggle to include even part of this complexity. Some address the dynamics of the atmospheres as a whole (3D) as subjected to rotation, or as patches of the surface (wind studies), compromising on the details of the composition and radiative/convective properties. Others solve the composition and radiative/convective properties, compromising on dynamical effects such as rotation. In this paper, we review existing model atmospheres for EGPs, and present the first high spatial resolution local (as opposed to global) 2/3D radiation hydrodynamic simulations of EGP atmospheres including dust cloud formation.
\end{abstract}

The SED of a Hot Jupiter is composed of thermal radiation left over from contraction and formation processes, stellar radiation reflected off the planetary surface, and stellar radiation absorbed by the stellar surface and reemitted redwards by thermal radiation. Planet-to-star flux contrast levels are found to be around 7 dex at the reflection peak (5000Åfor a G2 type star) and 5 dex at near-infrared wavelengths. Since this contrast is more favorable at near-IR and Infrared wavelengths, caracterisation studies of planetary candidates found by imaging use model atmospheres ignoring the effects of impinging radiation on the shape of their SED, and account for stellar irradiation by applying an achromatic correction factor. These models (in Allard et al. 2001) are readily available via a web simulator (http://phoenix.ens-lyon.fr/simulator), and tested for the study of brown dwarfs which cover a similar range of parameters (Teff, surface gravity) than for young planetary mass objects.

While the study of brown dwarfs can be enlightening for the study of planetary atmospheres - brown dwarf atmospheres are the site of an onset of dust cloud formation, strong rotation, and/or magnetic fields - their study thus far proceeded using only 1D static, often plane-parallel model atmospheres of various degrees of radiative transfer sophistication: Opacity Sampling vs. K-coefficient techniques, NLTE, photoionisation and photochemistry vs. Equilibrium Chemistry, stationary particle diffusion solutions, cloud models, assuming adiabaticity instead of using the Mixing Length Theory for convective mixing, etc.

For instance, Barman, Hauschildt \& Allard (2001), Barman et al. (2002), and Barman, Hauschildt \& Allard (2005) have explored the impact of dust cloud formation, NLTE and the photoionisation of sodium, and a reconstruction of the planetary surface with 1D static models, using the (at depth) entropy matching technique often used in a similar way for the study of binary stars in astrophysics. This technique allows to explore the orbital phase variations of the SED. These models led to the co-discovery of water vapor in the STIS spectro-photometric observations of HD209458 b (Barman 2007). 
A further leap in terms of detailed model atmospheres was recently achieved by Freytag et al. (2009a), who have modeled the atmospheres of VLMs, BDs, and PMOs by local 2D and 3D high-resolution Radiation HydroDynamic (RHD) simulations, including a cloud model and dynamical molecular transport as well as impinging radiation by a parent star. These models led to a stratospheric temperature inversion needed to explain the SED of Hot Jupiters, and predict dust clouds to form easily in the atmospheres of the CoRot planets, except perhaps for CoRot-3b where dust is expected to form cirrus-like clouds in the optically transparent upper layers (Freytag et al. 2009b). These models suggest the formation of gravity waves, temporal intensity variability, and dynamical mixing in brown dwarf and Hot Jupiter atmospheres. Further advance on this front requires therefore the systematic and consistent account of non-equilibrium chemistry. Thus far, only some species have been explored, often using rates extrapolated to the atmospheric conditions of brown dwarfs (Saumon et al. 2006).

Another issue is the strong radiation field to which are subjected Hot Jupiters, and which raises, among others, the question of the redistribution of the stellar light around the planetary surface: $i$ ) via radiation (requiring 3D radiative transfer solutions), and $i i$ ) via winds due to pressure contrasts and/or rotation (requiring hydrodynamical studies). The entropy matching technique led to a day (1995K)-to-night (500K) side temperature contrast of $1500 \mathrm{~K}$. Iro, Bézard \& Guillot (2005), using time-dependant 1D radiative transfer, have accounted for wind velocities corresponding to the expected rotational period of a gravitationally locked (with the star) exoplanet. However, the self-consistent account of rotation is a yet unsolved challenge for the understanding of brown dwarfs and planetary properties. Accounting for rotation, while neglecting the opacities and convection as a trade-off, led to a day (1700K)-to-night $(300 \mathrm{~K})$ contrasts of the order of $600 \mathrm{~K}$.

A further step was achieved by Fortney et al. (2006) who computed phase spectra based on the results of hydrodynamical wind studies (Cooper \& Showman 2006), themselves based on the radiative timescales of Iro, Bézard \& Guillot (2005). These simulations led to stratospheric winds which could explain the observed Spitzer 4 to $6 \mu \mathrm{m}$ flux excess. A review of dynamical models of Hot Jupiter atmospheres is available in Showman, Menou \& Cho (2007).

\section{References}

Allard, F., Hauschildt, P. H., Alexander, D. R., Tamanai, A., \& Schweitzer, A. 2001, ApJ 556, 357

Barman, T. 2007, ApJ (Letters) 661, L191

Barman, T. S., Hauschildt, P. H., \& Allard, F. 2001, ApJ 556, 885

Barman, T. S., Hauschildt P. H., \& Allard, F. 2005, ApJ 632, 1132

Barman, T. S., Hauschildt, P. H., Schweitzer, A., Stancill, P. C., Baron, E., \& Allard, F. 2002, ApJ (Letters) 569, L51

Cooper, C. S. \& Showman, A. P. 2006, ApJ 649, 1048

Fortney, J., Cooper, C., Showman, A., Marley, M., \& Freedman, R. 2006, ApJ 652, 746

Freytag, B., Allard, F., Ludwig, H.-G., Homeier, D., \& Steffen, M. 2009a, A\& $A$, in press

Freytag, B., Allard, F., Ludwig, H.-G., Homeier, D., \& Steffen, M. 2009b, Proceedings of the Conference "Molecules in the Atmospheres of Extrasolar Planets" held at Paris Observatory, November 19, 2008.

Hauschildt, P. H., Allard, F., \& Baron, E. 1999, ApJ 512, 377

Iro, N., Bézard, B., \& Guillot, T. 2005, A\& $A$ 436, 719

Saumon, D., Marley, M. S., Cushing, M. C., Leggett, S. K., Roellig, T. L., Lodders, K., \& Freedman, R. S. 2006, ApJ 647, 552

Showman, A. P., Menou, K., \& Cho, J. Y. K. 2008, ASP Conference Series Vol. 398, 419 